\title{
ESTIMACIÓN DE SEDIMENTOS EN LA CUENCA PILCOMAYO USANDO UN PRODUCTO DE PRECIPITACIÓN COMBINADO CON SENSORES ABORDO DE SATÉLITES
}

Jhonatan E. Ureña, Oliver C. Saavedra, Moisés Perales

\section{RESUMEN}

El objetivo de este estudio es la obtención de un producto de precipitación combinando sensores remotos con mediciones en tierra y explorar sus posibles aplicaciones para estimar caudal líquido y sólido. El presente estudio se concentra en la porción de la cuenca Pilcomayo en territorio boliviano. Se emplearon dos productos de precipitación con base satelital: GSMaP y CHIRPS. Empleando un ajuste iterativo de estos productos con pluviómetros se han obtenido dos productos denominados GS y $\mathrm{CH}$ respectivamente. Para realizar la validación de los productos generados se comparó con la grilla GMET de $5 \mathrm{~km}$ de resolución del Balance Hídrico Superficial de Bolivia (BHSB). Como se ha encontrado que el producto GS muestra un performance un poco mayor al de $\mathrm{CH}$, usando la precipitación combinada GS se ha procedido a estimar el caudal por subcuencas. Para este último proceso se ha utilizado los coeficientes de escorrentía reportado en el BHSB. Se ha logrado estimar el caudal líquido empleando el producto de precipitación combinado GS y coeficientes de escorrentía reportados en el BHSB. Primeramente, se ha estimado los caudales específicos por subcuenca para el periodo 2001-2015. Se ha evidenciado que las magnitudes de caudal líquido son mayores en la zona de los llanos y congruente con los valores de precipitación. Posteriormente se ha estimado el caudal líquido en las áreas de aporte de nueve estaciones hidrométricas para comparar con los caudales reportados en los 70s, 80s y 90s. Si bien son periodos diferentes de análisis, se ha notado consistencia de los caudales simulados con los reportados en la mitad de las estaciones. En la estación hidrométrica de Villamontes, que cubre $91 \%$ de la zona de estudio, se ha estimado 6310 millones de $\mathrm{m}^{3}$ de agua en promedio interanual para el periodo 2001-2015. Posteriormente se ha estimado el caudal sólido en las mismas estaciones. En Villamontes se ha estimado 159 millones de Toneladas al año de producción de sedimento. De esta manera en el presente estudio se ha aprovechado del producto de precipitación combinado por subcuenca para estimar la variación espacial del caudal líquido y sólido en la zona de estudio. Se ha notado la importancia de la estimación de la precipitación como variable clave de los procesos hidrológicos, que bien puede ser aún mejor aprovechado utilizando modelos hidrológicos distribuidos para convertir la precipitación en caudal y a su vez en estimar la producción de sedimentos en una cuenca.

Palabras Clave: Bolivia, Cuenca Pilcomayo, Cuenca de La Plata, Precipitación, Sedimentos, GSMaP, CHIRPS.

DOI: $10.23881 /$ idupbo.020.1-5i 